\title{
Evaluación de la calidad metodológica de la literatura en evaluación económica en salud en Colombia: una revisión sistemática
}

\author{
Sara Atehortúa1 , Mateo Ceballos², Carlos Felipe Gaviria¹, Aurelio Mejía ${ }^{2,3}$ \\ 1 Departamento de Economía y Grupo de Economía de la Salud, Facultad de Ciencias Económicas, Universidad \\ de Antioquia, Medellín, Colombia \\ 2 Grupo de Economía de la Salud, Facultad de Ciencias Económicas, Universidad de Antioquia, Medellín, \\ Colombia \\ ${ }^{3}$ Instituto de Investigaciones Médicas, Facultad de Medicina, Universidad de Antioquia. Medellín, Colombia
}

Introducción. La evaluación económica es una herramienta que contribuye a establecer prioridades en la toma de decisiones en salud. La calidad metodológica de los estudios realizados en Colombia no se ha evaluado de manera sistemática.

Objetivo. Valorar la calidad metodológica de las evaluaciones económicas en salud publicadas en Colombia.

Materiales y métodos. Se llevó a cabo una revisión sistemática de evaluaciones económicas completas (costos y efectividad de, al menos, dos programas o servicios de salud) referidas a un problema de decisión en salud de Colombia. La búsqueda se hizo en bases de datos internacionales y colombianas, y se complementó con búsqueda manual y consulta con autores. La síntesis de los datos se hizo en tablas que incluían información relevante sobre los estudios, y la calidad metodológica se evaluó con un instrumento prediseñado. Las búsquedas se hicieron entre enero y febrero de 2012.

Resultados. Se incluyeron 48 estudios. En la mayoría de los estudios se hace explícito el punto de vista, se describen las alternativas comparadas y se hace un análisis incremental (sic.). Más de la mitad de los trabajos presentan limitaciones en la especificación del horizonte temporal o la tasa de descuento, en la estimación y actualización de los beneficios en salud y los costos, así como en la consideración de cuestiones de equidad o implementación. El análisis de la incertidumbre resulta ser el punto más problemático.

Conclusiones. Las evaluaciones económicas en salud publicadas en Colombia han aumentado en los últimos años; sin embargo, se observa una gran heterogeneidad en los métodos, lo cual dificulta la comparación de los resultados y su uso para la toma de decisiones en salud. Es fundamental avanzar en la estandarización de los métodos, con el fin de contribuir a generar información de mejor calidad que permita apoyar las decisiones en el sistema de salud colombiano.

Palabras clave: economía médica, costos y análisis de costo, economía de la salud, literatura de revisión como asunto, estudios de evaluación como asunto, Colombia.

doi: http://dx.doi.org/10.7705/biomedica.v33i4.1464

Quality assessment of economic evaluations in health care in Colombia: a systematic review

Introduction: Economic evaluation is a tool that can provide useful information to decision-makers in health. The methodological quality of Colombian studies has not been assessed in a systematic way.

Objective: To assess the methodological quality of economic evaluations in health care in Colombia.

Materials and methods: Systematic review of economic studies that assessed both costs and effectiveness of at least two technologies related to a decision problem in Colombia. Search was performed in international and Colombian databases and was completed with manual searches and contact with authors. Data was synthesized in tables that included relevant information about the studies. Methodological quality was evaluated using a predefined instrument. Searches were performed between January and February.

Results: 48 studies were included in the review. Perspective of the study, incremental analyzes and description of alternatives were usually well specified. However, more than half of the articles

\section{Contribución de los autores:}

Sara Atehortúa fue la investigadora principal del proyecto.

Sara Atehortúa, Mateo Ceballos, Aurelio Mejía diseñaron y enviaron las encuestas.

Todos los autores participaron en la aplicación de los criterios de inclusión y exclusión a las referencias encontradas en bases de datos y otras fuentes, en la evaluación de la calidad metodológica, en la síntesis de los resultados, en la redacción de este manuscrito, así como en la revisión luego de recibir el concepto de los evaluadores. 
did not state clearly the time horizon or discount rate and most studies did not address equity and implementation issues. Management of uncertainty was also problematic.

Conclusions: Economic evaluation in health care in Colombia has grown considerably in recent years. However, methods vary considerably between studies and therefore their usefulness for decisionmaking in health is limited. It is necessary to standardize methods in order to generate evidence of higher quality to support decisions within the Colombian health care system.

Key words: Economics, medical; costs and cost analysis; health economics; review literature as topic; evaluation studies as topic; Colombia.

doi: http://dx.doi.org/10.7705/biomedica.v33i4.1464

En la actualidad, las sociedades experimentan un crecimiento exponencial de la tecnología médica, lo que ha permitido aumentar los beneficios potenciales en salud gracias a las alternativas cada vez más variadas y complejas, pero también aumentan los costos que estas tecnologías conllevan. De hecho, el gasto mundial promedio en salud (como porcentaje del PIB) ha crecido de $8,8 \%$ en 1995 a 10,3 \% en 2009 (1). En general, el incremento en los costos de estas nuevas tecnologías no se equipara con el aumento de los recursos disponibles para financiarlas (2).

Por ello, surge la necesidad de generar una mejor asignación de los recursos limitados, con el fin de alcanzar mayores niveles de bienestar y mejorar la satisfacción de las necesidades en salud (3). Un proceso explícito, basado en criterios relevantes, sobre cuáles tecnologías en salud financiar con recursos públicos, puede lograr mayor legitimidad por parte de los diferentes actores sociales. La evaluación económica de tecnologías y programas de salud surge como una rama de la economía de la salud, la cual pretende contribuir en dicha definición de prioridades. En términos generales, consiste en la identificación, medición y valoración de los costos y beneficios en salud de diferentes alternativas, y la elección de una o varias de ellas con base en la información disponible (4).

Siguiendo a Drummond (4), dos aspectos distinguen los tipos de evaluaciones económicas que pueden encontrarse en la literatura científica. Por un lado, están las evaluaciones parciales, que consideran los costos, consecuencias o ambas de sólo una alternativa de salud, o que comparan dos o más alternativas, pero sólo abordan sus costos o consecuencias. Por otro lado, están las

\footnotetext{
Correspondencia:

Sara Catalina Atehortúa, Facultad de Ciencias Económicas, Universidad de Antioquia, Calle 67 N 53-108, oficina 13-404, Medellín, Colombia

saritac2005@gmail.com, scatehortua@economicas.udea. edu.co
}

Recibido: 31/01/13; aceptado:26/06/13 evaluaciones económicas completas, las cuales comparan al menos dos alternativas y, además, consideran tanto sus costos como sus beneficios en salud.

Entre las evaluaciones económicas completas se pueden identificar tres tipos básicos de acuerdo con la forma de valorar los beneficios en salud. El análisis de costo-efectividad estima los beneficios en salud en unidades naturales (años de vida ganados, número de diagnósticos correctos 0 muertes evitadas, entre otros). El análisis de costoutilidad estima la efectividad en términos de años de vida ajustados por calidad, los cuales son un indicador que permite incorporar en una unidad el impacto que tiene un alternativa en salud sobre la esperanza y calidad de vida de los individuos. Tanto en el análisis de costo-efectividad como en el de costo-utilidad, el objetivo de la evaluación consiste en determinar la razón incremental (sic.) de costoefectividad; esto es, el costo adicional que implica generar una unidad adicional de beneficio en salud. El tercer tipo, es el análisis de costo-beneficio, en el cual se miden las consecuencias en términos monetarios para expresar el beneficio neto de un programa sobre otro (4), el cual ha sido el menos usado en la práctica.

Teniendo en cuenta el creciente desarrollo que ha tenido la evaluación económica en Colombia, no solo en términos de mayor número de publicaciones en los últimos años (5), sino también en los recientes intentos por incluir criterios técnicos para la toma de decisiones en salud del país, se justifica conocer con precisión la calidad metodológica de la investigación en este campo en Colombia, lo cual constituye el objetivo de este trabajo.

\section{Materiales y métodos}

\section{Tipo de estudio: revisión sistemática de la literatura}

Bases de datos consultadas: Health Technology Assessment Database y NHS Economic Evaluation Database (bases de datos especializadas que 
indizan evaluaciones económicas y reportes de evaluación de tecnologías incluidas en Medline, The Cochrane Library, EMBASE, PSYCINFO y CINAHL, entre otras; además de los reportes de las páginas de 52 miembros de la International Network of Agencies for Health Technology Assessment y otras 20 organizaciones de evaluaciones de tecnología en salud alrededor del mundo), Portal de Evidencias de la Biblioteca Virtual en Salud de la Organización Panamericana de la Salud (OPS) y Biblioteca Científica Electrónica en Línea (SciELO).

Para identificar estudios no publicados, se consultaron las bases de datos de borradores de trabajo en economía Research Papers in Economics y Dotec-Colombia, y se hizo una búsqueda libre en Scholar Google. También, se hizo una búsqueda manual en las revistas de medicina y economía registradas en las categorías $A 1, A 2, B$ y $C$ en la II Actualización del Índice Bibliográfico Nacional de Colombia (IBN Publindex) de 2010, y en las hojas de vida (CVLAC) de los autores identificados en las búsquedas. Además, se envió una encuesta a estos mismos autores para validar el listado de artículos identificados en la búsqueda y solicitar otras referencias no recuperadas mediante las búsquedas electrónica y manual.

Términos de búsqueda. En las bases de datos especializadas en evaluaciones económicas, se empleó el término Colombia (como término $\mathrm{MeSH}$ y descriptor libre truncado). En las bases de datos no especializadas, el término Colombia se combinó con términos como pharmacoeconomics, cost effectiveness, cost utility, cost benefit y sus equivalentes en español (cuadro 1).

Criterios de inclusión. Solo se incluyeron evaluaciones económicas completas referidas a un problema de decisión en salud de Colombia, es decir, estudios que analizaran tanto los costos como los beneficios en salud de al menos dos alternativas para solucionar un problema en salud específico, ya fuera para promoción, prevención, tamización, diagnóstico, tratamiento o rehabilitación de una enfermedad en Colombia.

Criterios de exclusión. Se excluyeron informes de caso, revisiones de evaluaciones económicas, opiniones de expertos publicadas como editoriales o cartas al editor, artículos metodológicos y estudios de minimización de costos o de costos de la enfermedad. No se aplicaron restricciones por idioma, fecha de publicación o nacionalidad de los autores. Las búsquedas se hicieron entre enero y febrero de 2012

Selección de artículos. Los criterios de inclusión y exclusión se aplicaron inicialmente a los títulos y resúmenes de las referencias identificadas. Posteriormente, se obtuvo el texto completo de los artículos que superaron este primer filtro y se aplicaron los mismos criterios al texto completo, para determinar finalmente cuáles se incluirían en la revisión. Esta selección se hizo de manera independiente por dos investigadores y todas las diferencias se solucionaron por consenso.

Síntesis de información: las principales características de los estudios se sintetizaron en tablas prediseñadas, las cuales incluían información sobre perspectiva del estudio, horizonte temporal, fuente de información para costos y efectividad, resultados y conclusiones de los autores, entre otros aspectos.

Evaluación de la calidad metodológica: a partir de los criterios establecidos por Drummond (4) y el Critical Appraisal Skills Programme en Español (6), se propuso una serie de ítems para evaluar el diseño metodológico y la presentación de resultados de los estudios. En el cuadro 2 se describen los criterios que fueron utilizados para esta evaluación, divididos en cuatro posibles respuestas:

Cuadro 1. Protocolo de búsqueda en bases de datos

Health Technology Assessment Database y NHS Economic Evaluation Database

\#1 MeSH DESCRIPTOR Colombia EXPLODE ALL TREES

\#2 colombia*

\#3 \#1 OR \#2

Portal de Evidencias de la Biblioteca Virtual en Salud - OPS:

Colombia* $^{*}$

SciELO, Research Papers in Economics (REPEC), Dotec-Colombia y Scholar Google:

("costo efectividad" OR "costo beneficio" OR "costo utilidad" OR "cost effectiveness" OR "cost utility" OR "cost benefit" OR

pharmacoeconom* OR farmacoeconom* OR "Technology assessment” OR "evaluación de tecnología”) AND colombia* 
Cuadro 2. Criterios para la evaluación de la calidad metodológica

Ítem

Punto de vista de la investigación

Descripción adecuada de las alternativas evaluadas

Efectividad

Costos

Actualización de costos y consecuencias futuros

Análisis incremental

Incertidumbre y análisis de sensibilidad

Otros aspectos relevantes para la decisión

\section{Criterios de evaluación}

S: Se menciona explícitamente la perspectiva adoptada, independientemente de cuál sea ésta. $\mathrm{N}$ : No se menciona explícitamente la perspectiva adoptada.

S: Se detallan las características de las intervenciones o programas por comparar; por ejemplo, dosis de medicamentos o duración de los tratamientos.

P: Sólo se presentan algunas características de las intervenciones, pero no todas las necesarias para los usuarios de la información; por ejemplo, no se especifican las dosis de los medicamentos. $\mathrm{N}$ : No se detallan adecuadamente las alternativas, simplemente se mencionan.

S: Para investigaciones basadas en la literatura se especifican claramente las bases de datos consultadas, términos de búsqueda y criterios de selección de los estudios que se seleccionan como fuentes de información. Para trabajos basados en datos de estudios primarios se describe adecuadamente el diseño del estudio.

P: Para estudios basados en la literatura sólo se presentan algunos criterios y justificaciones de la elección de los estudios; por ejemplo, se presentan las bases de datos consultadas pero no los términos de búsqueda. Para artículos basados en estudios primarios no se describen todas las características del diseño del estudio.

$\mathrm{N}$ : Para estudios basados en la literatura no se presenta ninguna justificación para la selección de los estudios (por ejemplo, sólo se menciona la referencia de donde provienen los datos). Para investigaciones basadas en datos primarios, sólo se menciona el estudio, sin hacer ninguna descripción.

S: Se detallan claramente los costos incluidos en el análisis, así como las fuentes a partir de las cuales de identificaron, midieron y valoraron los recursos consumidos.

P: Ofrece información parcial, por ejemplo, se detallan adecuadamente los costos incluidos, pero no se especifican correctamente las fuentes de información, o viceversa.

$\mathrm{N}$ : No se especifica con claridad cuales costos fueron considerados en la evaluación, y tampoco es posible saber con exactitud cuáles fueron las fuentes de información utilizadas.

S: Se especifica cuál fue la tasa de descuento utilizada.

$\mathrm{N}$ : No se menciona si se utiliza tasa de descuento, a pesar de considerar un horizonte temporal mayor de un año, o no es explícita un horizonte temporal.

NA: Para los artículos que tienen un horizonte temporal de un año o menos, no es necesario utilizar tasa de descuento.

S: En el estudio se hace un análisis comparativo de los costos y las consecuencias de las alternativas evaluadas. Para estrategias no dominadas, debe calcularse la relación incremental de costo-efectividad (para ACE y ACU) o el valor presente neto (para ACB).

$\mathrm{N}$ : A pesar de que se consideran tanto costos como consecuencias de las alternativas comparadas, no se hace un análisis incremental.

S: Se aborda la incertidumbre mediante un análisis de sensibilidad probabilístico que justifique los rangos de los parámetros del modelo, o mediante uno determinístico multivariado, y en el cual se justifiquen los rangos de variación de las variables.

$\mathrm{N}$ : Si en el trabajo no se presenta ningún tipo de análisis de sensibilidad, si no se justifican los rangos utilizados para modificar los parámetros, o si sólo se hace un análisis determinístico univariado.

S: En el trabajo se consideran los siguientes tres elementos: se hacen comparaciones con otros estudios similares y se exploran las razones de las posibles diferencias o similitudes en los resultados, se evalúan implicaciones de equidad y se consideran problemas de factibilidad de implementación.

P: En el trabajo sólo se considera alguno o algunos de los elementos anteriores, pero no todos. Por ejemplo, se hacen comparaciones, pero no se consideran cuestiones éticas ni de implementación; o se comparan y consideran problemas de implementación, pero no se tiene en cuenta ningún tipo de discusión de equidad.

$\mathrm{N}$ : En el trabajo no se considera ninguno de los tres elementos anteriores: no se hacen comparaciones con otros estudios (ni se menciona que no existen en la literatura otros trabajos que comparen las mismas alternativas), ni se tienen en cuenta cuestiones éticas o de implementación.

ACE: análisis de costo efectividad; ACU: análisis de costo-utilidad; ACB: análisis de costo-beneficio 
- se aborda adecuadamente el elemento considerado (S);

- se considera de manera parcial (P);

- no se analiza o aborda de manera adecuada $(\mathrm{N}), \mathrm{y}$

- no aplica hacer la calificación (NA)

La calidad de los estudios fue evaluada de manera independiente por los cuatro autores de esta investigación, y las diferencias se solucionaron por consenso.

\section{Resultados}

De 251 referencias potencialmente relevantes, se evaluó la calidad metodológica de 48 (7-54). En la figura 1 se presenta el flujograma del proceso de selección de estos estudios (55). De 68 encuestas enviadas para la validación de la búsqueda, se obtuvo respuesta en el 17,6 \% (12 cuestionarios diligenciados).

Las características generales de los estudios se presentan en el cuadro 3, agrupándolos bajo la Clasificación Internacional de Enfermedades (CIE 10) de la Organización Mundial de la Salud. El 77,1 $\%$ de los estudios corresponde a análisis de costoefectividad, el $18,8 \%$ son análisis de costo-utilidad, y el $4,2 \%$ restante se clasifican como análisis de costo-beneficio. En los últimos cinco años (20082012) se concentra el $83,3 \%$ de las publicaciones y el 16,7\% restante se publicó antes de 2008 (figura 2). El $68,8 \%$ se publicó en revistas nacionales y, el $31,3 \%$, restante en revistas internacionales. En el cuadro 4 se presentan las revistas en donde se han publicado estos artículos.
Referencias identificadas en las bases de datos $\mathbf{n}=\mathbf{2 2 8}$ Scielo/Publindex $n=124$

$\mathrm{CRD}=18$

Lilacs $=76$

Agencias iberoamericanas $=0$

Repec, Dotec y Scholar Google $=10$
Referencias identificadas en otras fuentes $\mathbf{n}=\mathbf{2 3}$

CVLAC de autores $=14$

Publindex de Colciencias $=6$

Encuesta de Investigadores $=3$
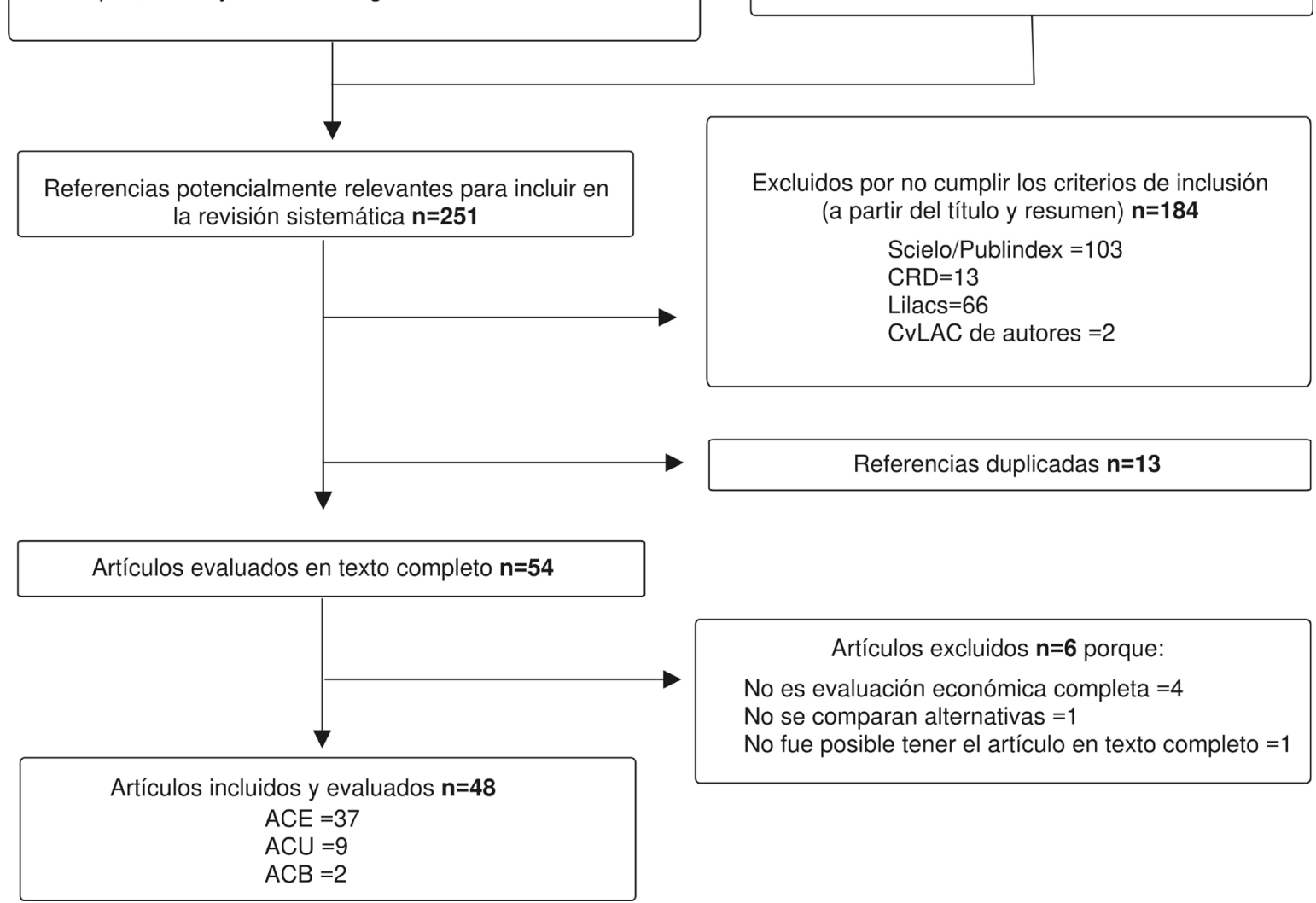

Artículos excluidos $\mathbf{n}=6$ porque:

No es evaluación económica completa $=4$ No se comparan alternativas $=1$

No fue posible tener el artículo en texto completo $=1$

Figura 1. Flujograma del proceso de selección de estudios. Adaptación del esquema propuesto por el grupo PRISMA ACE: análisis de costo efectividad; ACU: análisis de costo-utilidad; ACB: análisis de costo-beneficio 


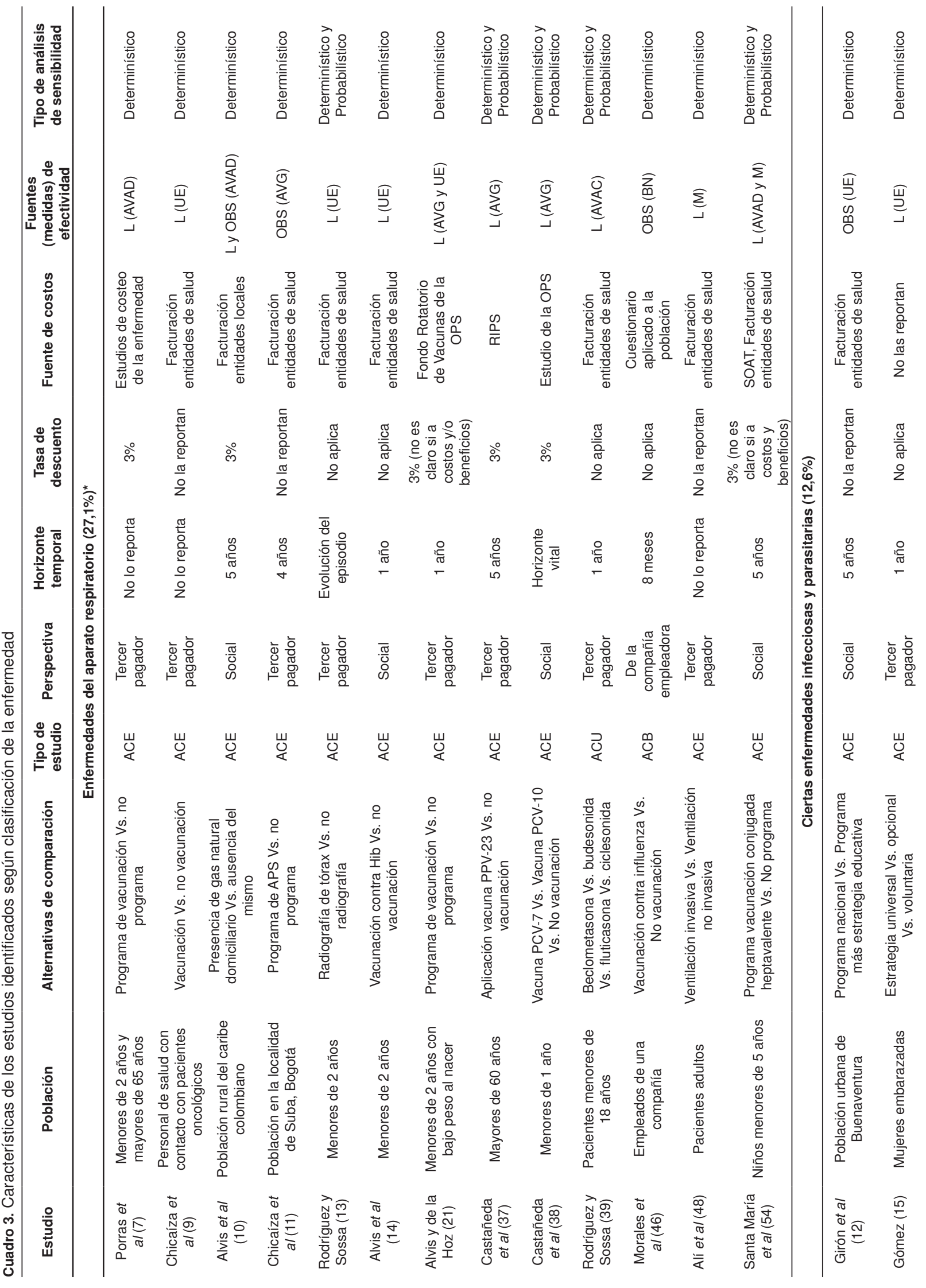



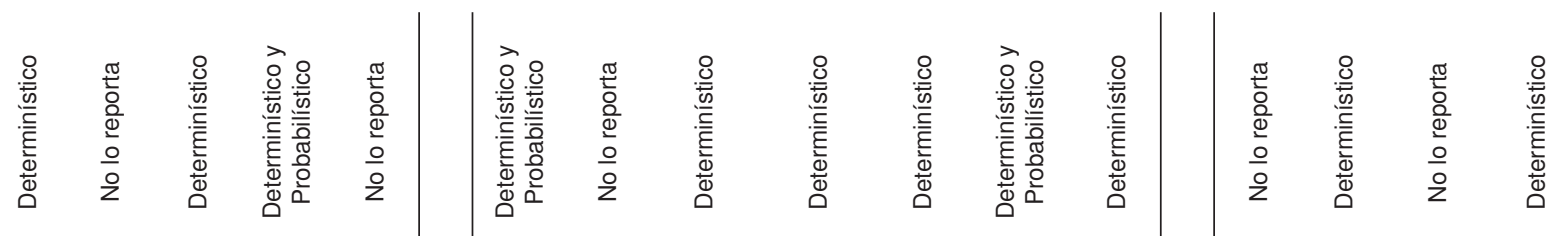

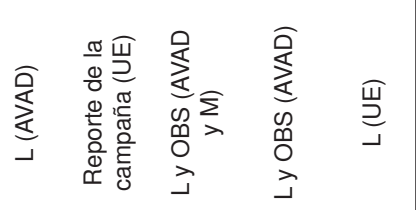

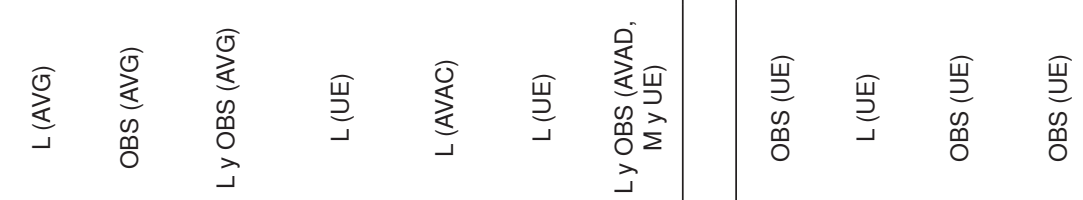

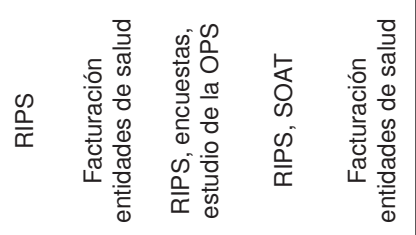

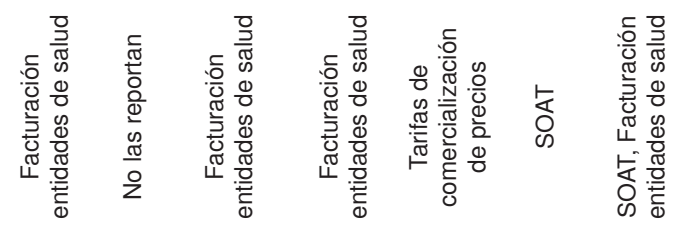

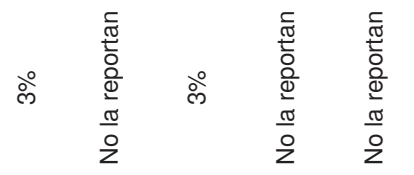

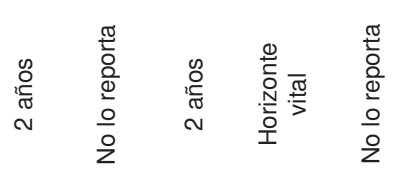

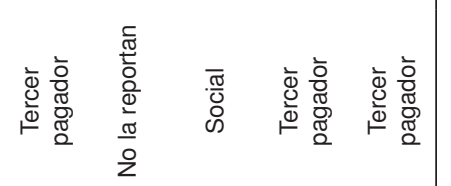

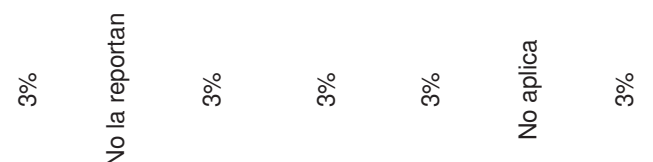

岁岸岸岸岸

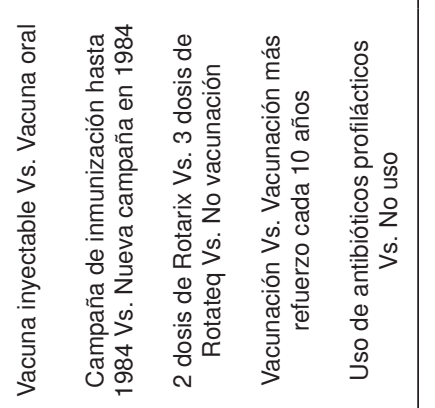

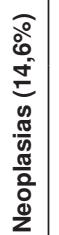

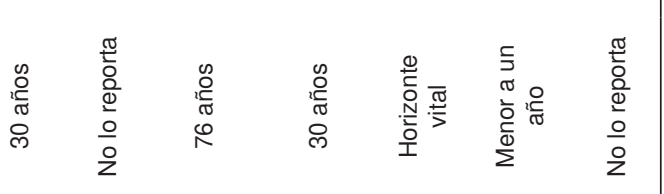

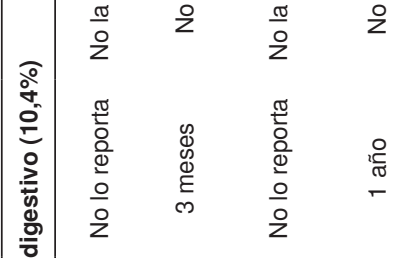

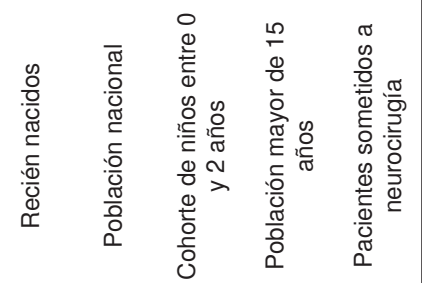

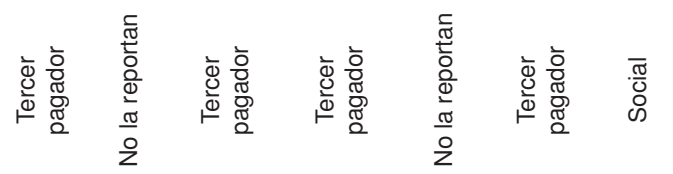

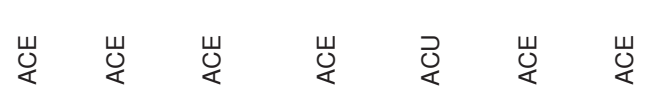
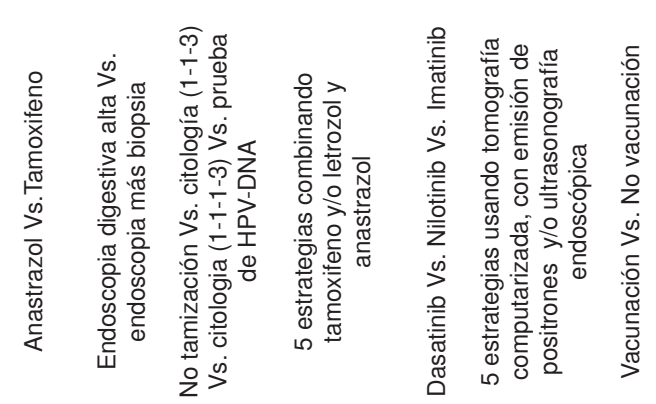

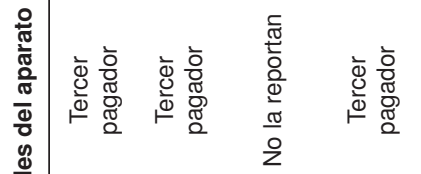

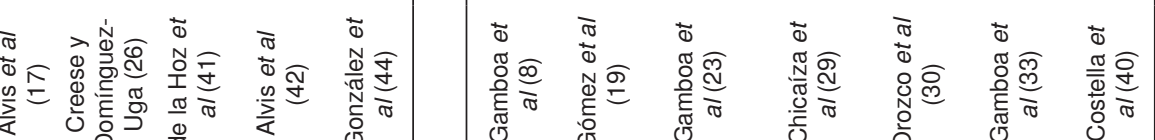

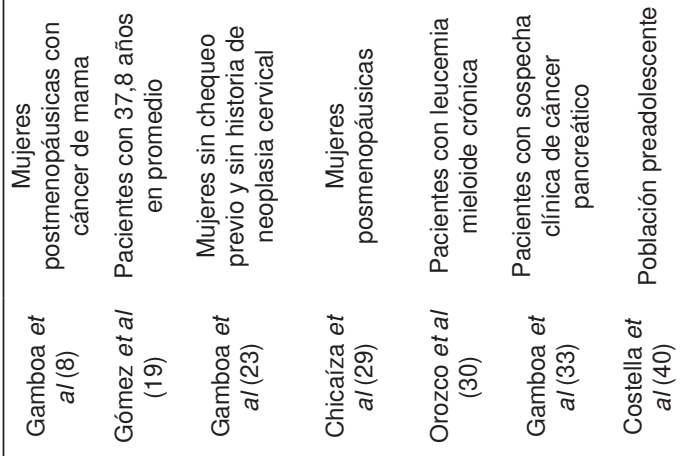

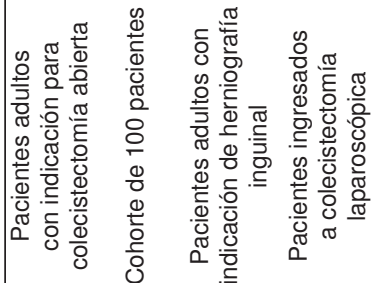

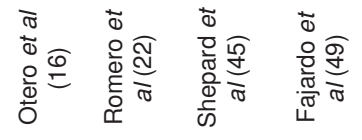




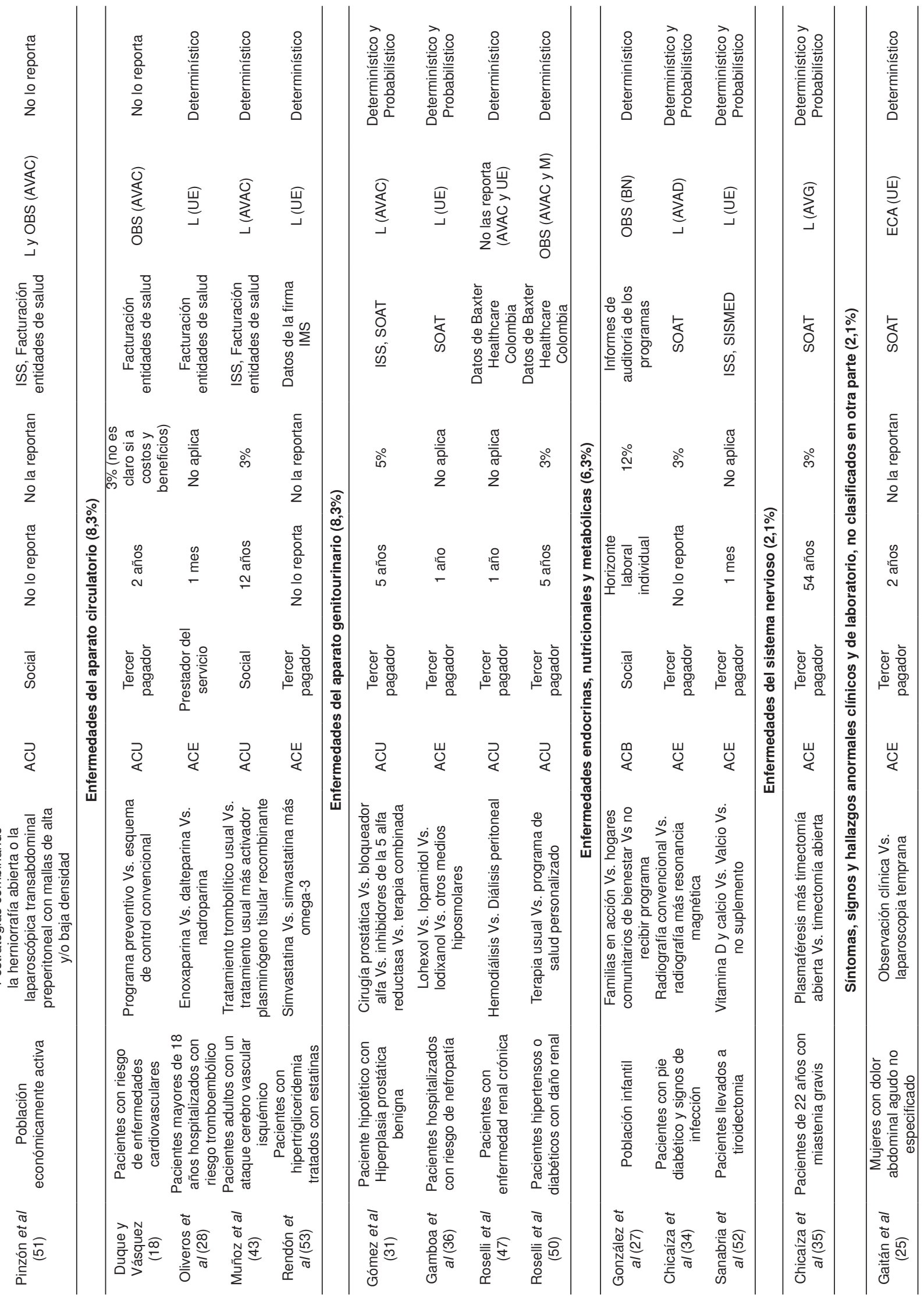




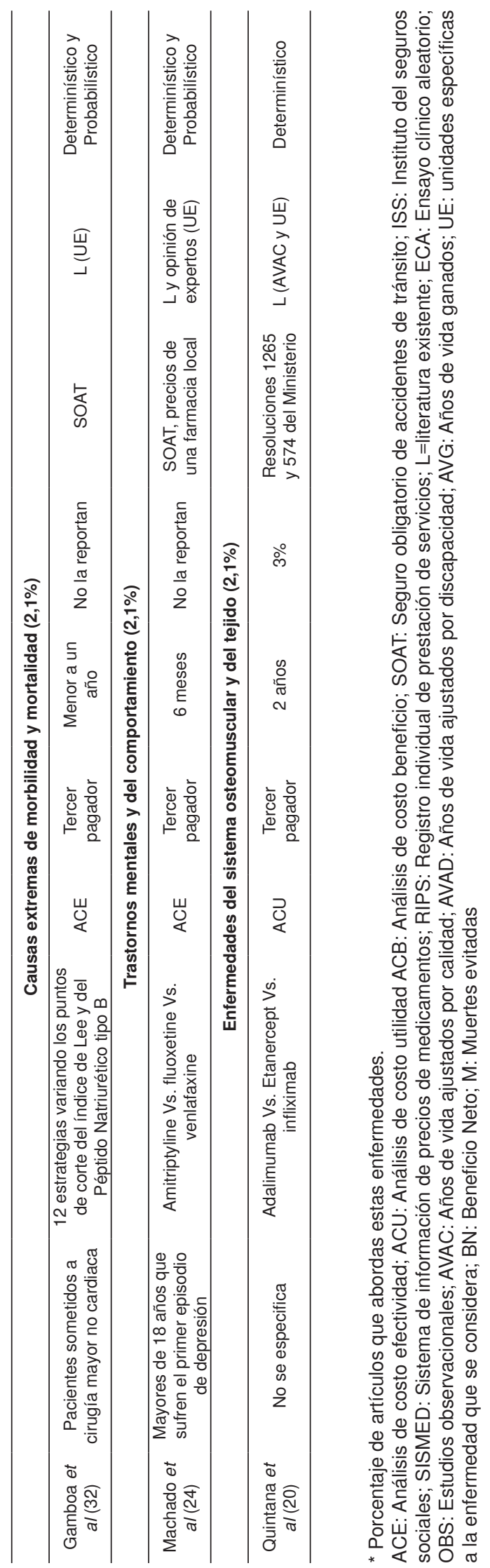




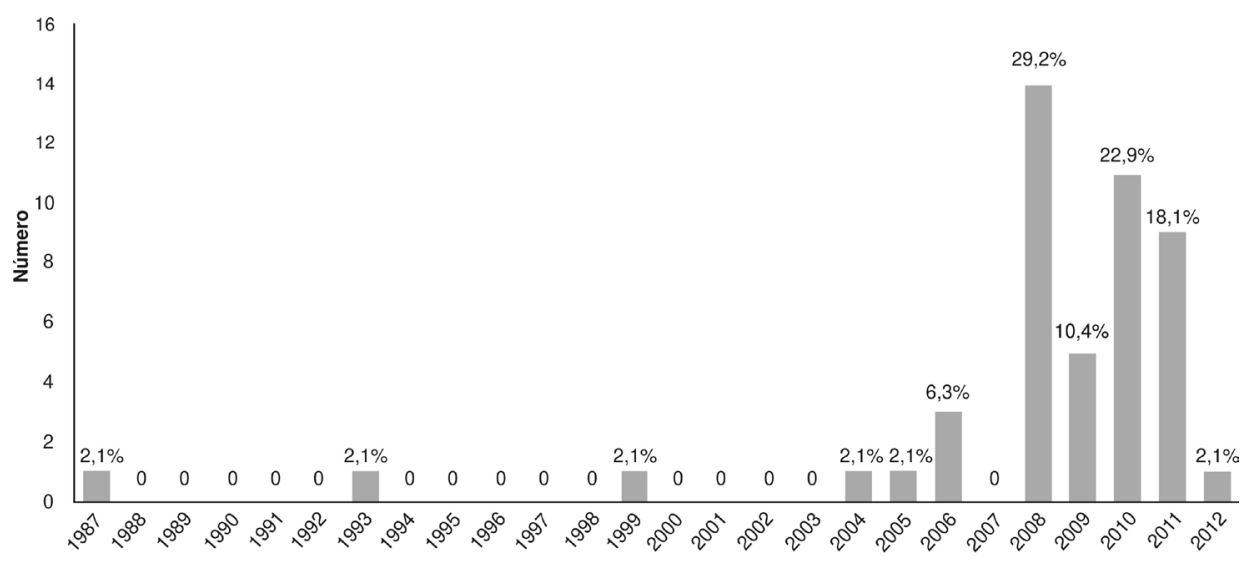

Figura 2. Distribución de los estudios según año de publicación

Cuadro 4. Distribución de los estudios según revista de publicación

\begin{tabular}{lcc}
\hline Revista & Número & Porcentaje \\
\hline Revista Panamericana de Salud Pública & 6 & 12,5 \\
Revista de Salud Pública & 6 & 12,5 \\
Borradores de trabajo de la Facultad de Ciencias Económicas & 5 & 10,4 \\
de la Universidad Nacional de Colombia & 4 & 8,3 \\
Biomédica & 3 & 6,3 \\
Vaccine & 2 & 4,2 \\
Value in Health & 10 & 20,8 \\
Revistas especializadas en diferentes enfermedades médicas & 12 & 25,0 \\
Otras revistas & 48 & 100,0 \\
Total & & \\
\hline
\end{tabular}

En $31,3 \%$ de los estudios se adopta un horizonte temporal menor o igual a un año; en $25 \%$ se contempla horizontes no mayores de cinco años; en $16,7 \%$ se considera un horizonte de largo plazo; y en otro $25 \%$ no se reporta ningún horizonte temporal. Los estudios confluyen en proponer tasas de descuento tanto para costos como para consecuencias del $3 \%$ : el 33,3\% de los trabajos lo reportan así y el $6,3 \%$, aunque también lo reportan, no especifican si se aplica a costos, a consecuencias o a ambos. En $27,1 \%$ de los estudios no se especifica ninguna tasa, ni se justifican las razones por las que no descuentan costos y beneficios futuros a pesar de emplear horizontes mayores a un año. En uno de los estudios evaluados se afirma utilizar una tasa de descuento de $3 \%$, aunque se explicita un horizonte temporal de un año.

En $50 \%$ de los estudios se utilizan medidas de efectividad específicas para cada enfermedad, en $18,8 \%$, años de vida ajustados por calidad, en $16,7 \%$, años de vida ajustados por discapacidad, en $16,7 \%$, años de vida ganados, en $10,4 \%$, muertes evitadas, y en $4,2 \%$ (correspondientes a los trabajos de análisis de costo-beneficio), unidades monetarias. En $72,9 \%$ de las referencias se usa información proveniente de la literatura para estimar la efectividad; en otro $33,3 \%$ se emplean estudios observacionales para este fin; en 4,2\% se recurre a otras fuentes; $y$ en $2,1 \%$ se extrajeron los datos de un ensayo clínico aleatorio realizado en Colombia.

Respecto a las fuentes para la estimación de costos, el $14,6 \%$ de los trabajos se basan en el Manual Tarifario del Instituto de Seguros Sociales (ISS); 8,3\%, en el Registro Individual de Prestación de Servicios de Salud (RIPS); 25 \%, en el Manual del Seguro Obligatorio de Accidentes de Tránsito (SOAT); 2,1 \%, en el Sistema de Información de Precios de Medicamentos (SISMED); 43,8 \%, en registros y facturaciones de diferentes entidades de salud (como institutos, hospitales o empresas promotoras de salud) y el $25 \%$ utiliza otras fuentes.

El análisis de sensibilidad determinístico es el más usado en los estudios, pues en $54,2 \%$ se recurre a éste para modelar la posible incertidumbre existente en las estimaciones; en 31,3\%, además del determinístico, también se emplea el análisis 
Cuadro 5. Evaluación de la calidad metodológica

\begin{tabular}{|c|c|c|c|c|c|c|c|c|}
\hline & & & & & Ítem & & & \\
\hline Estudio & $\begin{array}{l}\text { Punto } \\
\text { de vista }\end{array}$ & $\begin{array}{c}\text { Descripción } \\
\text { adecuada de } \\
\text { las alternativas }\end{array}$ & Efectividad & Costos & $\begin{array}{c}\text { Actualización } \\
\text { de costos y } \\
\text { consecuencias } \\
\text { futuras }\end{array}$ & $\begin{array}{c}\text { Análisis } \\
\text { incremental }\end{array}$ & $\begin{array}{l}\text { Incertidumbre } \\
\text { y análisis de } \\
\text { sensibilidad }\end{array}$ & $\begin{array}{l}\text { Otros aspectos } \\
\text { relevantes } \\
\text { para la decisión }\end{array}$ \\
\hline
\end{tabular}

Enfermedades del aparato respiratorio

Porras, et al. (7)

Chicaíza, et al. (9)

Alvis, et al. (10)

Chicaíza, et al. (11)

Rodríguez y Sossa (13)

Alvis, et al. (14)

Alvis y de la $\mathrm{Hoz}(21)$

Castañeda, et al. (37)

Castañeda, et al. (38)

Rodríguez y Sossa (39)

Morales, et al. (46)

Alí, et al. (48) S

Santa María, et al. (54)

S
S
S
S
S
S
S
S
S
S
S
N
S

S
S
S
S
S
S
$S$
$S$
$S$
$S$
$P$
$P$
$S$

Ciertas enfermedades infecciosas y parasitarias

Girón, et al. (12)

Gómez (15) S

Alvis, et al. (17)

Creese y Domínguez (26)

De la Hoz, et al. (41)

Alvis, et al. (42)

González, et al. (44)

S

Neoplasias

Gamboa, et al. (8)

Gómez, et al. (19)

Gamboa, et al. (23)

Chicaíza, et al. (29)

Orozco, et al. (30)

Gamboa, et al. (33)

Costella, et al. (40)

Enfermedades del aparato digestivo

Otero, et al. (16)

Romero, et al. (22)

Shepard, et al. (45)

Fajardo, et al. (49)

Pinzón, et al. (51)

$\mathrm{S}$
$\mathrm{N}$
$\mathrm{S}$
$\mathrm{S}$
$\mathrm{S}$

$\begin{array}{llll}S & S & P & N\end{array}$

Enfermedades del aparato circulatorio

Duque y Vásquez (18)

Oliveros, et al. (28)

Muñoz, et al. (43)

Rendón, et al. (53)

Enfermedades del aparato genitourinario

Gómez, et al. (31)

Gamboa, et al. (36)

Roselli, et al. (47)

Roselli, et al. (50)

S
S
S
N
S
S

Enfermedades endocrinas, nutricionales y metabólicas

González, et al. (27)

Chicaíza, et al. (34)

Sanabria, et al. (52)

$\begin{array}{ll}\text { S } & \text { S } \\ \text { S } & P \\ N & P \\ \text { S } & N \\ \text { S } & P\end{array}$

sistema ne

$\begin{array}{ll}S & S \\ S & S \\ S & S \\ S & S\end{array}$

Chicaíza,

Síntomas, signos y hallazgos anormales clínicos y de laboratorio, no clasificados en otra parte

Gaitán, et al. (25) S S S $\mathrm{S}$

Gamboa, et al. (32) S P
Trastornos mentales y del comportamiento

Machado, et al. (24)

$\mathrm{S}$

\author{
$P \quad S$
}

Enfermedades del sistema osteomuscular y de tejidos blandos

Quintana, et al. (20)

$\mathrm{S}$

$P \quad S$

$\mathrm{N} \quad \mathrm{S} \quad \mathrm{N}$

$\mathrm{N} \quad \mathrm{S}$

P NA

SA

$S$

S N

S: sí; P: parcialmente; N: no; NA: no aplica 
probabilístico; y en $14,6 \%$ no hace o no se reporta ningún tipo de análisis.

En el cuadro 5 se reportan los resultados de la evaluación de la calidad metodológica. A continuación se mencionan los ítems en los cuales buena parte de los artículos reciben un concepto favorable. En primer lugar, la definición explícita del punto de vista, en el cual en $64,6 \%$ de los artículos se adopta una perspectiva del tercer pagador $y$, en $20,8 \%$, una perspectiva social; en $6,3 \%$ se abordan otras perspectivas, como el punto de vista del empleador en una empresa privada y solamente en $8,3 \%$ no se detalla la perspectiva asumida. En segundo lugar, la mayoría de las investigaciones hace un análisis incremental y sólo el 14,6 \% (todos correspondientes a análisis de costo-efectividad y de costo-utilidad) no lo realiza. En tercer lugar, en la generalidad de los artículos se describen detalladamente las alternativas comparadas, ya que en $67 \%$ de los trabajos se hace adecuadamente.

En los demás aspectos del diseño y presentación de resultados, se observan limitaciones importantes. Con respecto a la adecuada estimación de la efectividad, solo en $40 \%$ de los estudios se presentan de manera detallada la metodología y las fuentes empleadas para la estimación de la efectividad. En $54 \%$ de los trabajos se provee información de manera parcial sobre la estimación de los costos, pues en algunos se reportan de manera detallada los costos incluidos pero no se presentan de manera explícita las fuentes empleadas para su valoración, o viceversa. La actualización de costos y consecuencias futuras también presenta dificultades, ya que en $27 \%$ de los estudios no se utiliza la tasa de descuento, a pesar de adoptar un horizonte temporal mayor de un año y no se justifica dicha decisión.

El ítem con mayores deficiencias es el análisis adecuado de la incertidumbre. Si bien en un gran porcentaje de los artículos se hace el análisis de sensibilidad, solo en $25 \%$ se presenta adecuadamente y se justifican los rangos de los parámetros usados. El último ítem de calificación corresponde a la discusión de otros aspectos relevantes para la decisión, en el cual se observa que en $31 \%$ de los trabajos se discuten cuestiones relacionadas con la factibilidad de implementación de la alternativa propuesta; apenas en $29 \%$ se abordan cuestiones éticas e implicaciones de equidad; y en $52 \%$ de los trabajos se comparan sus resultados con estudios similares y se exploran las posibles razones de las diferencias.

\section{Discusión}

EnColombia, lostrabajos publicadossobreevaluación económica han crecido considerablemente en los últimos años (5). Las fortalezas de los artículos identificados radican en: la descripción adecuada de las alternativas, lo que brinda claridad sobre qué es lo que se está evaluando; en la presentación adecuada de los resultados de acuerdo con las prácticas establecidas en la literatura científica internacional (4), la cual permite la comparación de resultados entre estudios en contextos en donde existe una regla de decisión clara; y, en la especificación de la perspectiva, porque es posible saber para quiénes se está desarrollando la evaluación.

Las limitaciones de los artículos se asocian con: la no especificación del horizonte temporal, que impide determinar si se contemplan todos los beneficios y costos relevantes para las alternativas; la falta de modelado a largo plazo en los casos en los que era posible; la carencia del uso de tasas de descuento en estudios con un horizonte temporal mayor de un año, porque la estimación de costos y consecuencias no resulta adecuada; la falta de claridad de las fuentes de información utilizadas, debido a que disminuye la confiabilidad de los resultados; la falta de un abordaje adecuado de la incertidumbre, lo que implica que no se considera la variabilidad en los resultados; $y$, que no se incluyen otros aspectos relevantes como cuestiones éticas o de factibilidad de implementación, elementos que serían importantes para los tomadores de decisiones en salud.

Los resultados de este estudio son coherentes con los reportados por una revisión sistemática y evaluación de la calidad metodológica de evaluaciones económicas realizadas entre 1980 y 2004 en América Latina y el Caribe (56), en la cual se concluyó que dicha calidad en la región es pobre y heterogénea, fallando sobre todo en la especificación de fuentes de información, perspectiva y análisis incremental, entre otros. Por otro lado, en una revisión sistemática de evaluaciones económicas en España (entre 1983 y 2008), también se resaltó la heterogeneidad de la calidad de las fuentes de información reportada sobre métodos de análisis, fuentes de datos y tipos de intervención (57).

En este estudio se ofrece una revisión sistemática de la literatura científica existente en evaluación económica en salud en Colombia, con una búsqueda amplia y rigurosa que disminuye al 
máximo la posibilidad de sesgo de publicación y que además evalúa la calidad metodológica de esta literatura, contribuyendo en la formulación de recomendaciones para homogenizar métodos y facilitar que la evaluación económica se convierta en un insumo útil para los tomadores de decisiones. Entre las limitaciones del estudio se encuentran la dificultad para acceder a todos los estudios llevados a cabo en Colombia, pues es posible que muchas evaluaciones económicas sean resultado de consultorías, asesorías o ambas para la empresa privada y en tal sentido no se incentiva $o$ no se permite su publicación. Las pesquisas manuales siempre tienen la posibilidad de omisión de algún artículo no identificado, lo cual es problemático ya que: los CVLAC de los autores no siempre están actualizados y hace falta claridad en la información que allí se reporta; el instrumento de medición de la calidad metodológica empleado no es estandarizado, ni contextualizado al caso colombiano; se obtienen pocas respuestas por parte de los investigadores a las encuestas enviadas.

Las implicaciones de los resultados de este estudio apuntan a que hay dificultades en el uso de las evaluaciones económicas hechas en Colombia para la toma de decisiones en salud, y en la comparación de sus resultados, debido a la gran heterogeneidad en los métodos y en la forma de reportar los resultados. Es fundamental avanzar en la estandarización de los métodos con el fin de contribuir a generar información de mayor calidad que permita apoyar las decisiones en el sistema de salud colombiano. Cuestiones como la correcta especificación de quién se asume como tercer pagador, el abordaje de una perspectiva social, la explicitación de los criterios para identificar y seleccionar fuentes de información de costos y consecuencias, y la realización adecuada del análisis de sensibilidad, son algunos de los elementos metodológicos que podrían contribuir en dicha estandarización, pero además, el establecimiento de una guía metodológica colombiana (58) puede minimizar la heterogeneidad metodológica existente.

Particularmente, se recomienda que los análisis de sensibilidad no se limiten a uno determinístico univariado, debido a que por lo general los rangos son arbitrarios y no se tiene en cuenta las posibles correlaciones entre las variables (59). Siguiendo a Claxton (59) y Briggs (60), se recomienda complementar el análisis determinístico con uno probabilístico, el cual permite analizar de forma más completa la incertidumbre en el modelo. En todos los tipos de análisis es fundamental justificar los rangos que se utilizaron, ya que los resultados dependen en gran medida de ello. Finalmente, un elemento adicional que se recomienda, que en los últimos años ha cobrado importancia en las discusiones metodológicas a nivel internacional y que sólo se aborda en uno de los trabajos (39), es el análisis del valor esperado de la información perfecta, herramienta que permite hacer una estimación del valor que una sociedad podría estar dispuesta a pagar por realizar más investigación que disminuyera la incertidumbre $(60,61)$.

Como focos de desarrollo futuros de esta investigación, podría considerarse la construcción de un instrumento más estandarizado para la evaluación de la calidad metodológica, como ha hecho la epidemiología clínica con las lecturas críticas de la calidad de la evidencia. Obviamente esto requiere, por lo menos en el contexto colombiano, de una comunidad académica consolidada que participe en la elaboración y discusión de tal instrumento. Además de lo anterior, podría considerarse un análisis de conflictos de intereses y su relación con la calidad metodológica de los trabajos.

\section{Agradecimientos}

Los autores agradecen a Diana Londoño y María Elena Mejía por sus aportes en la búsqueda, a los investigadores que participaron en el diligenciamiento de la encuesta y a los evaluadores externos de la revista. Cualquier error u omisión es responsabilidad de los autores.

\section{Conflicto de intereses}

Los autores declaran que no existieron conflictos de interés al momento de la realización de la investigación.

\section{Financiación}

Este trabajo es resultado de la investigación "Estado de la investigación en evaluación económica en salud en Colombia: análisis teórico y metodológico de la producción académica", realizada con el apoyo del Centro de Investigaciones y Consultorías - CIC- de la Facultad de Ciencias Económicas y de la Estrategia de Sostenibilidad 2011-2012 del Grupo de Economía de la Salud de la Universidad de Antioquia.

\section{Referencias}

1. Banco Mundial. Datos de libre acceso, 2012. Fecha de consulta: 9 de junio de 2012. Disponible en: http://datos. bancomundial.org/frontpage 
2. Fuchs VR. El futuro de la economía de la salud. Lecturas de Economía. 2001;55:8-30.

3. Ortún-Rubio V, Pinto-Prades J, Puig-Junoy J. El establecimiento de prioridades. Aten Primaria. 2001; 27:673-6.

4. Drummond MF. Methods for the economic evaluation of health care programmes. Oxford: Oxford University; 2005. p. 379.

5. Restrepo Zea JH, Atehortúa Becerra SC, Rodríguez Acosta SM. Introducción a la economía de la salud en Colombia. Medellín: Universidad de Antioquia; 2008. p. 269.

6. CASPe. Herramientas para el análisis crítico de literatura científica. Alicante: CASPe; 2010. Fecha de consulta: 25 de septiembre de 2012. Disponible en: http://redcaspe.org/ drupal/?q=node/29

7. Porras-Ramírez A, Alvis-Guzmán N, Rico-Mendoza A, Alvis-Estrada L, Castañeda-Orjuela CA, VelandiaGonzález MP, et al. Costo efectividad de la vacunación contra influenza en menores de 2 años y mayores de 65 años en Colombia. Rev Salud Pública. 2009;11:689-99. http://dx.doi.org/10.1590/S0124-00642009000500002

8. Gamboa O, Díaz S, Chicaíza L, García M. Análisis de costoefectividad en Colombia de anastrazol Vs. tamoxifeno como terapia inicial en mujeres con cáncer temprano de mama y receptor hormonal positivo. Biomédica. 2010;30:46-55.

9. Chicaíza-Becerra LA, García-Molina M, Ballesteros M, Gamboa O, Díaz J, Vega R. Evaluación económica de la vacuna contra la influenza aplicada al personal de salud que atiende pacientes oncológicos hospitalizados. Rev Salud Pública. 2008;10:759-66. http://dx.doi.org/10.1590/ S0124-00642008000500008

10. Alvis-Guzmán N, Alvis-Estrada L, Orozco-Africano J. Costo efectividad del gas natural domiciliario como tecnología sanitaria en localidades rurales del caribe colombiano. Rev Salud Pública. 2008;10:537-49. http:// dx.doi.org/10.1590/S0124-00642008000400004

11. Chicaíza L, García M. Evaluación económica y sostenibilidad financiera del Modelo de Atención Primaria en Salud (APS) en la localidad de Suba de Bogotá - Colombia. Rev Gerenc Polit Salud. 2008;7:110-24.

12. Girón SL, Mateus JC, Castellar CE. Análisis de costoefectividad de dos intervenciones para el control de la malaria en el área urbana de Buenaventura, Colombia. Biomédica. 2006;26:379-86.

13. Rodríguez CE, Sossa MP. Costo-efectividad de la radiografía de tórax en lactantes con sospecha clínica de bronquiolitis viral en Colombia. Rev Panam Salud Pública. 2011;29:153-61. http://dx.doi.org/10.1590/S102049892011000300002

14. Alvis N, De La Hoz F, Vivas D. Relación costo-efectividad de la vacuna contra Haemophilus influenzae tipo b en niños menores de dos años de edad en Colombia. Rev Panam Salud Pública. 2006;20:248-55. http://dx.doi.org/10.1590/ S1020-49892006000900005

15. Gómez M. Comparación de tres estrategias de tamizaje para la prevención de la infección perinatal por $\mathrm{VIH}$ en Colombia: análisis de decisiones. Rev Panam Salud Pública. 2008;24:256-64. http://dx.doi.org/10.1590/S102049892008001000005
16. Otero AJ, Alvis N, Palomino R. Análisis costo-efectividad del uso de la técnica anestésica subaracnoidea comparada con la técnica anestésica general para colecistectomía abierta. Rev Colomb Anestesiol. 2008;36:179-85.

17. Alvis N, De la Hoz F, Narváez J. Impacto económico de la introducción de la vacuna inactivada inyectable contra la poliomielitis en Colombia. Rev Salud Pública. 2010;27:352-9. http://dx.doi.org/10.1590/S1020-49892010000500005

18. Duque SL, Vásquez J. Riesgo cardiovascular, calidad de vida y años de vida ajustados por calidad: un estudio de caso. Invest Educ Enferm. 2010;28:32-42.

19. Gómez MA, Ricaurte O, Gutiérrez O. Costo efectividad de la endoscopia digestiva alta como prueba diagnóstica en una campaña para detección del cáncer gástrico. Rev Colomb Gastroenterol. 2009;24:34-50.

20. Quintana G, Restrepo JP, Cáceres H, Rueda JD, Rosselli D. Evaluación económica del tratamiento de artritis reumatoide con terapia biológica anti TNF en Colombia. Acta Med Colomb. 2011;36:24-9.

21. Alvis N, De la Hoz F. Cost effectiveness of heptavalent pneumococcal conjugate vaccine in populations of high risk in Colombia. Colomb Méd. 2010;41:315-22.

22. Romero J, Sanabria A, Angarita M, Varón JC. Costeffectiveness of computed tomography and ultrasound in the diagnosis of appendicitis. Biomédica. 2008;28:139-47.

23. Andrés-Gamboa O, Chicaíza L, García-Molina M, Díaz J, González M, Murillo R, et al. Cost-effectiveness of conventional cytology and HPV DNA testing for cervical cancer screening in Colombia. Salud Pública Mex. 2008;50:276-85.

24. Machado M, Lopera MM, Díaz-Rojas J, Jaramillo LE, Ray Einarson T. Pharmacoeconomics of antidepressants in moderate-to-severe depressive disorder in Colombia. Rev Panam Salud Pública. 2008;24:233-9. http://dx.doi. org/10.1590/S1020-49892008001000002

25. Gaitán H, Eslava-Schmalbach J, Gómez P. Cost effectiveness of diagnostic laparoscopy in reproductive aged females suffering from non-specific acute low abdominal pain. Rev Salud Pública. 2005;7:166-79. http://dx.doi. org/10.1590/S0124-00642005000200005

26. Creese AL, Domínguez-Uga MA. Cost-effectiveness of immunization programs in Colombia. Bull Pan Am Health Organ. 1987;21:377-94.

27. González LM, Claros SP, Cano G, Flechas H. Comparación evaluación costo-beneficio Programas Nutricionales en Colombia: Familias en acción y Hogares comunitarios. Archivos Economía. Fecha de consulta: 12 de septiembre de 2012. Disponible en: http://ideas.repec. org/p/col/000118/005501.html

28. Oliveros H, Lobero R, Martínez F. Análisis costo-efectividad de las heparinas de bajo peso molecular en trombo profilaxis temprana en pacientes médicos y quirúrgicos. Acta Med Colomb. 2006;31:71-82.

29. Chicaíza L, García M, Gamboa O. Tamoxifen vs inhibidores de aromatasa para cáncer de mama: análisis de costo efectividad para Colombia. Bogotá: Facultad de Ciencias Económicas, Universidad Nacional de Colombia; 2008.

30. Orozco JJ, Valencia JE, Aiello E, Ribón G, Guerrero $\mathbf{F}$, García R, et al. Costo efectividad del dasatinib en el 
tratamiento de la leucemia mieloide crónica en pacientes resistentes al imatinib. CES Medicina. 2010;24:31-45.

31. Gómez P, Plata M, Carreño A, Guzmán F, Jiménez LA, Calderón $\mathbf{C}$, et al. Evaluación económica del tratamiento de la hiperplasia prostática benigna en Colombia. Rev Urol Colomb. 2010;19:69-76.

32. Gamboa O, Chicaíza L, García M. Costo-efectividad de añadir el péptido natriurético tipo b al índice de Lee en la evaluación del riesgo cardiovascular en cirugía mayor no cardiaca. Bogotá: Universidad Nacional de Colombia. Facultad de Ciencias Económicas; 2011.

33. Gamboa O, Chicaíza L, García M. Cost-effectiveness of CT, EUS and PET/CT in evaluating patients with symptoms of pancreatic cancer in Colombia. Bogotá: Facultad de Ciencias Económicas, Universidad Nacional de Colombia; 2010.

34. Chicaíza-Becerra L, Gamboa O, García M. Evaluación de costo-efectividad de la adición de la resonancia magnética a la radiografía convencional en la evaluación de pacientes con pie diabético y signos de infección. Rev Salud Pública. 2010;12:974-81. http://dx.doi.org/10.1590/S012400642010000600009

35. Chicaíza L, García M, Gamboa O, Castañeda C. Costo efectividad de realizar plasmaféresis previa timectomía abierta en el tratamiento de la miastenia gravis. Bogotá: Universidad Nacional de Colombia, Facultad de Ciencias Económicas; 2011.

36. Gamboa O, García M, Chicaíza L. Costo-efectividad de los medios de contraste isoosmolares e hiposmolares en pacientes con riesgo elevado de nefropatía inducida por medios de contraste. Biomédica; 2012;32:182-8. http:// dx.doi.org/10.7705/biomedica.v32i2.367

37. Castañeda C, Alvis NR, Paternina A, De La Hoz F. Costeffectiveness of the introduction of the pneumococcal polysaccharide vaccine in elderly Colombian population. Vaccine.2011;29:7644-50.http://dx.doi.org/10.1016/j. vaccine.2011.08.006

38. Castañeda C, Alvis NR, Velandia M, De La Hoz F. Costeffectiveness of pneumococcal conjugate vaccines of 7,10 , and 13 valences in Colombian children. Vaccine. 2012;30:1936-43. http://dx.doi.org/10.1016/j.vaccine. 2012.01.031

39. Rodríguez CE, Sossa MP. Análisis de costo-utilidad de los esteroides inhalados disponibles en Colombia para el manejo de pacientes pediátricos con asma persistente. Rev Colomb Neumol. 2009;21:111-35.

40. Costella D, Goldie S, Alvis N, Oshea M, Sweet S, Valenzuela M, et al. Burden of human papillomavirus (HPV) infection and HPV related disease in Latin America and the Caribbean, and health and economic outcomes of HPV vaccination in selected countries in Latin America. Atlanta: U.S. Centers for Disease Control and Prevention; 2008.

41. De la Hoz F, Alvis N, Narváez J, Cediel N, Gamboa O, Velandia M. Potential epidemiological and economical impact of two rotavirus vaccines in Colombia. Vaccine. 2010;28:3856-64.http://dx.doi.org/10.1016/j.vaccine. 2010.03.004

42. Alvis N, De la Hoz F, Gamboa O, Cediel N, Rico A, Paternina A. Impacto epidemiológico y económico de la vacunación contra el tétanos en adultos de Colombia.
Rev Panam Salud Pública. 2011;30:209-16. http://dx.doi. org/10.1590/S1020-49892011000900004

43. Muñoz M, Gutiérrez AM, Londoño D, Bayona H, Herrán S, Pérez GE. Uso del activador de plasminógeno tisular recombinante (rt-PA) en el ataque cerebrovascular isquémico (ACVi) en Colombia: un estudio de costo-efectividad. Acta Neurol Colomb. 2008;24:158-73.

44. González LF, Osorio E, Londoño D, Dennis R. Costo efectividad del uso profiláctico de antibióticos en Neurocirugía. Univ Med. 1999;40:43-8.

45. Shepard DS, Walsh J, Munar W, Rose L, Guerrero R, Cruz LF, et al. Cost-effectiveness of ambulatory surgery in Cali, Colombia. Health Policy Plan. 1993;8:136-42. http:// dx.doi.org/10.1093/heapol/8.2.136

46. Morales A, Martínez MM, Tasset-Tisseau A, Rey E, Baron-Papillon F, Follet A. Cost and benefits of influenza vaccination and work productivity in a Colombian company from the employer's perspective. Value Health. 2004;7:43341. http://dx.doi.org/10.1111/j.1524-4733.2004.74006.x

47. Rosselli D, De Antonio R, Calderón C. Análisis económico de diálisis peritoneal comparada con hemodiálisis en pacientes con enfermedad renal crónica, diabética 0 hipertensiva. Medunab. 2008;11:201-5.

48. Alí A, Cubillos L, Varón F, Soto J. Análisis de costo efectividad: ventilación invasiva frente a no invasiva en EPOC. Acta Colombiana de Cuidado Intensivo. 2010; 10:244-52.

49. Fajardo R, Valenzuela Jl, Olaya SC, Quintero G, Carrasquilla G, Pinzón CE, et al. Costo-efectividad de colecistectomía laparoscópica versus abierta en una muestra de población colombiana. Biomédica. 2011;31:51424.

50. Rosselli D, De Antonio R, Calderón C. Costo-efectividad de un programa de salud renal en pacientes con enfermedad renal crónica diabética y/o hipertensiva. Hospitalaria. 2008;10:12-8.

51. Pinzón FE, Rincón FM, Espitia E, Domínguez LC. Herniorrafia inguinal abierta versus laparoscópica y con nuevos materiales protésicos: análisis de su efectividad clínica y económica para el paciente, el hospital y el sistema sanitario. Rev Colom Cir. 2011;26:242-59.

52. Sanabria A, Domínguez LC, Vega V, Osorio C, Duarte D. Cost-effectiveness analysis regarding postoperative administration of vitamin-D and calcium after thyroidectomy to prevent hypocalcaemia. Rev Salud Pública 2011;13:80413. http://dx.doi.org/10.1590/S0124-00642011000500009

53. Rendón DA, Ordoñez JE, Orozco JJ. Costo-efectividad de los ácidos grasos omega 3 como coadyuvante en hipertrigliceridemia. Value Health. 2011;14:545-6. http:// dx.doi.org/10.1016/j.jval.2011.08.1581

54. Santamaría M, García F, Uribe MJ. Evaluación económica de la inclusión de la vacuna antineumocócica en el Plan Ampliado de Inmunización. Bogotá: Fedesarrollo; 2008. p. 84.

55. Urrutia G, Bonfill X. Declaración PRISMA: una propuesta para mejorar la publicación de revisiones sistemáticas y meta análisis - Elsevier. Medicina Clínica. 2010;135:507-11.

56. Augustovski F, Iglesias C, Manca A, Drummond M, Rubinstein A, Marti SG. Barriers to generalizability of 
health economic evaluations in Latin America and the Caribbean region. Pharmacoeconomics. 2009;27:919-29. http://dx.doi.org/10.2165/11313670-000000000-00000.

57. Catalá-López F, García-Altés A. Evaluación económica de intervenciones sanitarias en España durante el período 1983-2008. Rev Esp Salud Pública. 2010;84:353-69.

58. Ministerio de la Protección Social. Guía Metodológica para la elaboración de Guías Atención Integral en el Sistema General de Seguridad Social en Salud Colombiano. Bogotá, 2010. Fecha de consulta: 9 de junio de 2012. Disponible en: http://www.minsalud.gov.co/salud/Documents/Gu\%C3\%
ADa\%20Metodol\%C3\%B3gica\%20para\%20la\%20 elaboraci\%C3\%B3n\%20de\%20gu\%C3\%ADas.pdf

59. Claxton K. Exploring uncertainty in cost-effectiveness analysis. Pharmacoeconomics. 2008;26:781-98.

60. Briggs AH, Claxton K, Sculpher MJ. Decision modeling for health economic evaluation. Oxford: Oxford University; 2006. p. 237.

61. Claxton K, Sculpher M, Drummond M. A rational framework for decision making by the National Institute for Clinical. Lancet. 2002;360:711-5. http://dx.doi.org/10.1016/ S0140-6736(02)09832-X 